\title{
MicroRNA-199a Upregulation Mediates Lumbar Intervertebral Disc Degeneration and is Associated with Clinical Grades of Degeneration
}

\author{
Majid Reza FARROKHI ${ }^{1}$, Mohammad Hossein KARIMI ${ }^{2}$, Fariborz GHAFFARPASAND ${ }^{3}$, Masih SHERAFATIAN ${ }^{4}$ \\ ${ }^{1}$ Shiraz University of Medical Sciences, School of Medicine, Shiraz Neuroscience Research Center, Department of Neurosurgery, Shiraz, Iran \\ ${ }^{2}$ Shiraz University of Medical Sciences, Shiraz Transplant Research Center, Shiraz, Iran \\ ${ }^{3}$ Shiraz University of Medical Sciences, Research Center for Neuromodulation and Pain, Shiraz, Iran \\ ${ }^{4}$ Tarbiat Modares University, Faculty of Biological Sciences, Department of Molecular Genetics, Tehran, Iran
}

This study has been presented at the $26^{\text {th }}$ International Student Congress of (bio) Medical Sciences (ISCOMS 2019) between 3 and 7 June 2019 at Leiden, Netherlands.

Corresponding author: Fariborz GHAFFARPASAND fariborz.ghaffarpasand@gmail.com

\section{ABSTRACT}

AIM: To determine the expression profile of miRNA-199a-5p in intervertebral disc degeneration (IDD) and its correlation to the grade of IDD.

MATERIAL and METHODS: This case-controlled study was conducted during a 6-month period from 2017 to 2018 in two university hospitals in Shiraz, Iran. We included 15 patients with grade 3 and 4 of Pfirrmann and 5 patients with traumatic lumbosacral fractures with grade I. Total discectomy was performed in all the individuals and the samples were sent to the laboratory. The nucleus pulposus (NP) cells were isolated and the RNA was extracted. cDNA was synthesized by reverse transcriptase and the expression was measured using real-time polymerase chain reaction (RT-PCT).

RESULTS: We overall included 20 patients in two study groups. Both study groups were comparable regarding the baseline and clinical characteristics except for age $(p=0.026)$. The fold change $(p=0.007)$, and relative expression $(p=0.012)$ of the miRNA-199a$5 p$ was found to be significantly higher in patients compared to controls. The fold change $(p=0.001)$, and relative expression $(p<0.001)$ were also associated with the Pfirrmann grading. We found that the area under curve (AUC) was 0.880 (95\% Cl: $0.721-$ 0.938) indicative of moderate accuracy.

CONCLUSION: Expression of the miRNA-199a-5p is increased in the IDD. The expression of the miRNA-199a-5p was also associated with the grade of the degeneration based on the Pfirmann grading.

KEYWORDS: Intervertebral disc degeneration, MicroRNA-199a, Target genes, Pfirrmann grade

\section{INTRODUCTION}

I ntervertebral disc degeneration (IDD) is currently considered the etiology of low back pain (LBP) which is the secLond most common complain of the patients referring to the outpatient clinics worldwide (12). LBP is associated with high disease burden and disability worldwide. According to the global burden of the disease, LBP ranked highest in terms of disability (YLDs), and sixth in terms of overall burden (DALYs) with a global prevalence of $9.4 \%$ in 2010 , increasing with age (6). There are several key steps identified in IDD which includes loss of extracellular matrix, endplate cartilages hyperplasia and subsequent sclerosis, loss of height and release of pro-inflammatory cytokines $(19,24)$. The nucleus pulposus (NP) cells are cartilage-like cells with minimal regenerative
Majid Reza FARROKHI (1) : 0000-0002-4949-7623 Mohammad Hossein KARIMI (D) : 0000-0002-2435-6277
Fariborz GHAFFARPASAND (1) : 0000-0002-1721-9987 Masih SHERAFATIAN O : 0000-0002-1101-1966 
potentials which maintain the intervertebral disc function and structure (1). The apoptosis and senescence of the NP cells is considered the key step in IDD and understanding the factors contributing to NP cell apoptosis will shed light on the pathogenesis of IDD and also the possible treatment options $(2,3)$. Recently, the role of micro-RNAs (miRNA) in regulation of cell-mediated transcription of human genome has been investigated which has resulted in understanding of many mechanisms development of new treatment modalities (27). These molecules are non-coding small-size RNA which have 20-22 nucleotides in length and are produced from different sections of the genome $(26,27)$. Several recent studies have investigated the expression profile of miRNAs in IDD and has found the association between various types and different key steps in IDD including cell proliferation, lineage determination, apoptosis, cytokine release, as well as extracellular matrix (ECM) anabolism and catabolism $(8,10)$. In a recent meta-analysis of three major microarray studies, we have clearly demonstrated that miRNA-199a-5p is upregulated in IDDand mediates the pathogenesis of the IDD throughECM-receptor interaction, adherens junction, Hippo signaling pathway and TGF-beta signaling pathway (16). The miRNA-199a-5p target the COL1A1, COL6A1, and COL5A1 and thus dysregulates the synthesis of the collagen fibers in the NP and AF (16). The proposed pathway for this has been demonstrated to be the Hippo pathway which regulates the contact inhibition, and cell senescence leading to intervertebral disk degeneration (28). However, complementary study is required to shed light on the issue. Thus, the aim of the current study was to determine the quantitative expression of the miRNA-199a-5p in lumbar IDD. We also aimed to determine the proposed pathway and the target gene of the aforementioned miRNA.

\section{MATERIAL and METHODS}

\section{Study Population}

This case-controlled study was conducted during a 6-month period from December 2017 to June 2018 in Shahid Chamran hospital, a tertiary healthcare center and referral center of spine surgery, and Emtiaz hospital a level I trauma center, both affiliated with Shiraz University of Medical Sciences. We have included a total number of 15 adult (>18 years) patients with degenerative lumbar intervertebral disc disease who were scheduled for discectomy. The degeneration of the intervertebral disc was defined according to the Pfirrmann grading system (14). We included only those with Pfirrmann grades of III and IV as patients. Those with Pfirrmann grades of I, II and V, those receiving high doses of corticosteroids before surgery and those on anticoagulants were excluded from the study. We also included a total number of 5 adult $(>18$ years) patients with normal intervertebral discs according to the Pfirmann grading as the control group. We only included those with grade I degeneration and excluded those with grade II. All the controls were selected from those referring to our trauma center with lumbosacral fractures requiring surgery. We have excluded all the patients and the controls who had any history of malignancy or previous spine surgery. The study protocol was approved by the institutional review board (IRB) and medical ethics committee of Shiraz University of Medical Sciences (IR.SUMS.MED.REC.1396.s390). All the patients and the controls provided their informed written consents before inclusion in the study.

\section{Study Protocol}

All the patients were evaluated for eligibility based on the history, physical examination and T2-weighted MRI. The patients were initially evaluated by the neurosurgery attending physician and neurosurgery resident and a complete history and physical examination was performed. The findings were recorded into the data gathering forms. We recorded the demographics (age, gender, location of residence), past medical history (hypertension, diabetes mellitus, smoking, opium addiction and thyroid diseases), clinical signs (radiculopathy, myelopathy, motor or sensory deficits and sphincter function), and characteristics of the IDD (level of the IVD and the Pfirmann grade). All the patients then underwent surgery and the disc specimen was obtained and transferred to the Transplant Research Center of Shiraz University of Medical Sciences for further analysis. All the samples were immediately placed in RNA later stabilization solution being manufactured in Shiraz Pharmacy School. The samples were then frozen at $-20^{\circ} \mathrm{C}$ for further evaluation and assessment.

\section{Isolation of the NP Cells}

The NP cells were extracted according to the instructions described previously (7). First the samples were completely homogenized using the homogenizer machine (Merck, Germany). Briefly, NP tissue specimens were washed twice with Phosphate Buffered Saline (PBS), then minced and digested with $2 \mathrm{U} / \mathrm{mL}$ protease (Gibco, Grand island NY, USA) for 30 minutes at $37^{\circ} \mathrm{C}$. NP cells were released from the tissue by treating with $0.25 \mathrm{mg} / \mathrm{mL}$ type II collagenase (Gibco, Cat. No. 17101-015) for 4 hours at $37^{\circ} \mathrm{C}$. The resulting cell suspension was transferred into a $40 \mu \mathrm{m}$ cell strainer (Novin Daru, Tehran, Iran) and centrifuged at $800 \mathrm{~g}$ for 5 minutes. The NP cells were resuspended in the medium containing $10 \%$ fetal bovine serum (FBS, Gibco, Grand island NY, USA), $100 \mathrm{U} /$ $\mathrm{mL}$ penicillin, $100 \mu \mathrm{g} / \mathrm{mL}$ streptomycin, and $1 \%$ L-glutamine. Cells were incubated at $37^{\circ} \mathrm{C}$ in $5 \% \mathrm{CO}_{2}$ and the medium was changed every 3 days.

\section{RNA Isolation and Reverse Transcription}

All the samples were stored again in $-20^{\circ} \mathrm{C}$ for the RNA extraction step. The RNA was extracted from the human NP samples using Trizol (Invitrogen, Cleveland, USA) according to the manufacturer's instructions. Concentration of total RNA was measured at $260 \mathrm{~nm}$ with a spectrophotometer (DU-800; Beckman Coulter, Brea, CA). First strand complementary DNA (cDNA) synthesis was performed with 500 ng of total RNA in a $10 \mu \mathrm{L}$ final volume containing $2 \mu \mathrm{L}$ Primer Script RT Master Mix (Takara, RR036A, Japan) and $8 \mu \mathrm{L}$ of RNase-free $\mathrm{dH}_{2} \mathrm{O}$ and total RNA. The reverse transcription procedure was carried out according to the manufacturer's instructions.

\section{Quantitative Real-Time Polymerase Chain Reaction}

Real-time PCR for miRNA-199a-5p and U6 was performed by using SYBR premix Ex Taq ${ }^{\mathrm{TM}}$ (Takara Bio Inc., Shiga, 
Japan) with a Step One Plus real-time PCR system (Applied Biosystems, Foster City, CA), according to the manufacturer's instructions. U6 was used to normalize the gene expression of mRNAs. The relative amount of transcripts was calculated according to the comparative CT method. All of the primers were synthesized by Invitrogen according to the manufactures instructions.

\section{Statistical Analysis}

The sample size calculation was performed according to the previous studies on IDD (7). Briefly, in order to detect at least $33 \%$ difference in relative expression of the tested miRNA compared to the housekeeping gene with a sensitivity of $80 \%$ and an error probability of $5 \%$, at least 5 patients were required in each groups. The results of the RT-PCR was analyzed using the CT analysis technique. The fold change and relative expression of the miRNA-199a-5p was calculated according to the subsequent equations:

$$
\begin{aligned}
& \text { Fold Change }=2^{-\triangle \triangle C t} \\
& \text { Relative Expression }=2^{-\Delta C t}
\end{aligned}
$$

The Statistical Package for Social Science, SPSS for Windows, version 22.0 (SPSS Inc., Chicago, ILL., USA) was used for data analysis. The proportions were compared using the chi-square test between the two study groups. the parametric variable with normal distribution were compared using the independent t-test between two study groups and using One-way analysis of variance (ANOVA) for more than 2 groups using the LSD as post hoc test. The parametric variables without normal distribution were compared using the Mann-Whitney $U$ and Kruskal-Wallis test were appropriate. A univariate Spearsman correlation analysis was also performed to detect linear correlation between corresponding variables. The receiver-operative characteristic ( $\mathrm{ROC}$ ) curve analysis was also done to determine the sensitivity of the miRNA199a$5 p$ in detection for the IDD. A 2-sided p-value of less than 0.05 was considered statistically significant.

\section{RESULTS}

\section{Baseline Characteristics}

A total of 20 patients were included in the current study. We included 15 patients with grades III and IV of Pfirrmann and 5 patients with grade I Pfirrmann. All the patients underwent disc sampling in two of our centers. The mean age of the patients was significantly higher than controls $(p=0.026)$. There were 7 $(46.7 \%)$ men and $8(53.3 \%)$ women among the patients while there were $4(80.0 \%)$ men and $1(20.0 \%)$ women among the controls. There were no statistically difference between the two study groups regarding the gender distribution $(p=0.319)$. The baseline characteristics of the patients is summarized in Table I. The most common level of involvement was L4/L5 in both study groups. There was no significant difference between the two study groups regarding the level of involvement $(p=0.108)$. Those in the patient group had significantly higher frequency of radiculopathy when compared to the controls $(p=0.009)$. The clinical signs and symptoms along with the Pfirrmann grading in two study groups is demonstrated in Table II.

\section{Expression of miRNA-199a-5p}

We demonstrated that the fold change of the miRNA-199a$5 p$ was significantly higher in patients when compared to the controls $(p=0.007)$ (Figure 1A). The relative expression of the miRNA-199a-5p was significantly higher in patients when compared to the controls $(p=0.012)$ (Figure 1B). We found that the fold change was significantly higher in grade IV when compared to the grade I $(p=0.001)$ but there was no significant difference between group I and III $(p=0.202)$. The fold expression was also higher in grade IV compared to grade III ( $p=0.017)$ (Figure $1 \mathrm{C})$. In the same way, the relative expression was significantly higher in grade IV when compared to the grade I $(p<0.001)$ but there was no significant difference between group I and III ( $p=0.339)$. The fold expression was also higher in grade IV compared to grade III $(p=0.003)$ (Figure 1D).

Table I: The Baseline Characteristics of the 15 Patients with Disc Degeneration and 5 Controls with Traumatic Injuries Included in the Current Study

\begin{tabular}{lccc}
\hline & $\begin{array}{c}\text { Patients } \\
(\mathbf{n = 1 5 )}\end{array}$ & $\begin{array}{c}\text { Controls } \\
(\mathbf{n = 5})\end{array}$ & $\mathbf{p}$ \\
\hline Age (years) & $48.80 \pm 13.4$ & $32.71 \pm 10.3$ & $\mathbf{0 . 0 2 6}$ \\
\hline Gender & & & 0.319 \\
\hline Men (\%) & $7(46.7)$ & $4(80.0)$ & $1(20.0)$ \\
\hline Women (\%) & $8(53.3)$ & & 0.766 \\
\hline Comorbidities & & $1(20.0)$ & 0.530 \\
\hline Smoking (\%) & $4(26.7)$ & $0(0.0)$ & 0.389 \\
\hline Hypertension (\%) & $0(0.0)$ & 0.389 \\
\hline CAD (\%) & $4(26.7)$ & $0(0.0)$ & \\
\hline Asthma (\%) & $2(13.3)$ & & \\
\hline
\end{tabular}

CAD: Coronary artery disease. 
Table II: The Clinical Findings of the 15 Patients with Disc Degeneration and 5 Controls with Traumatic Injuries Included in the Current Study

\begin{tabular}{lccc}
\hline & $\begin{array}{c}\text { Patients } \\
(\mathbf{n}=\mathbf{1 5})\end{array}$ & $\begin{array}{c}\text { Controls } \\
(\mathbf{n}=5)\end{array}$ & $\mathbf{p}$ \\
\hline Level & & & 0.108 \\
\hline L3/L4 (\%) & $1(6.7)$ & $2(40.0)$ & \\
\hline L4/L5 (\%) & $9(60.0)$ & $3(60.0)$ & \\
\hline L5/S1 (\%) & $5(33.3)$ & $0(0.0)$ & $\mathbf{0 . 0 0 9}$ \\
\hline Clinic & & 0.055 \\
\hline Radiculopathy (\%) & $15(100.0)$ & $2(40.0)$ & 0.539 \\
\hline Axial LBP (\%) & $8(53.3)$ & $0(0.0)$ & 0.999 \\
\hline Claudication (\%) & $3(20.0)$ & $0(0.0)$ & 0.109 \\
\hline Paresthesia (\%) & $9(60.0)$ & $4(60.0)$ & 0.127 \\
\hline Sensory deficit (\%) & $4(26.7)$ & $4(80.0)$ & $<0.001$ \\
\hline Motor deficit (\%) & $5(33.3)$ & $4(80.0)$ & \\
\hline Pfirrmann Grade & & $5(100.0)$ & \\
\hline I (\%) & $0(0.0)$ & $0(0.0)$ & \\
\hline III (\%) & $5(33.3)$ & $0(0.0)$ & \\
\hline IV (\%) & $10(66.7)$ & & \\
\hline
\end{tabular}

LBP: Low back pain.

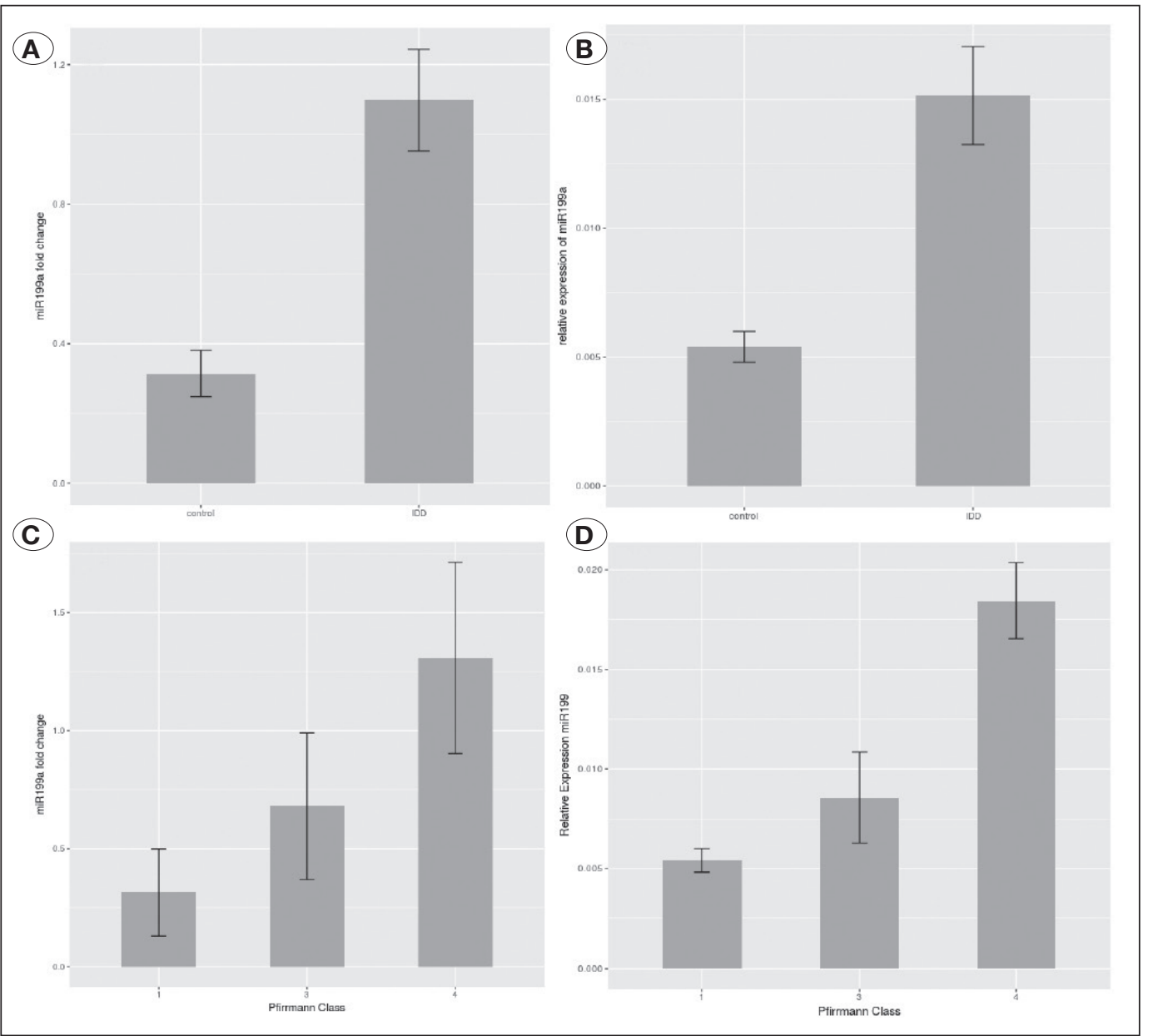

Figure 1: Fold change of miRNA199a-5p between patients and controls. As demonstrated the fold change is significantly higher in IDD patients $(p=0.007)$ (A); relative expression of miRNA199a-5p between patients and controls. As demonstrated the fold change is significantly higher in IDD patients $(p=0.012(B)$; fold change of miRNA199a-5p between different Pfirrmann grading (C); relative expression of miRNA199a-5p between different Pfirrmann grading (D). 


\section{Diagnostic Accuracy of miRNA-199a-5p}

We have evaluated the diagnostic accuracy of miRNA-199a$5 p$ as a biological marker of the IDD. For this purpose, the sensitivity multiply by $1 /$ specificity was calculated and the ROC curve was evaluated. The area under curve (AUC) was also calculated to determine the accuracy of the miRNA-199a$5 p$ fold change and relative expression. We found that the AUC was 0.880 (95\% Cl: $0.721-0.938)$ indicative of moderate accuracy test to diagnose the IDD (Figure 2).

\section{Functional Analysis and Pathway Annotation}

Four enriched KEGG pathways were 'fatty acid biosynthesis' (hsa00061), 'proteoglycans in cancer' (hsa05205), and 'fatty acid metabolism' (hsa01212). Most important targeted genes by hsa-miR-199a were identified using TarBase database (version 7) as fatty acid synthase (FASN) and stearoyl-CoA desaturase (SCD) in fatty acid metabolism pathway, and, 24-dehydrocholesterol reductase (DHCR24) and Cytochrome P450 family 51 subfamilies A member 1 (CYP51A1) in steroid biosynthesis pathway (Table III). Enrichment analysis of the Gene ontology (GO) function and KEGG pathways for miRNA-199a-5p were performed with a significance threshold

Table III: The KEEG Pathway of the miRNA-199a-5p in IDD

\begin{tabular}{lc}
\hline KEEG Pathways & $\mathbf{p}$ \\
\hline Fatty acid biosynthesis & 1.5765 \\
\hline Proteoglycans in cancer & 1.3394 \\
\hline Fatty acid metabolism & 5.1731 \\
\hline Steroid biosynthesis & 0.0010 \\
\hline
\end{tabular}



Figure 2: Receiver-operative characteristics (ROC) curve of the miRNA-199a-5p for detecting the IDD using the fold change and relative expression. AUC was equal to 0.880 (95\% Cl: $0.721-0.938)$ indicative of moderate accuracy. of less than 0.05. The most significant GO terms under the biological processes category were 'cellular nitrogen compound metabolic process' (GO:0034641), 'biosynthetic process' (GO:0009058) and 'cellular protein modification process' (GO:0006464). The most enriched GO terms under cellular components category were 'organelle' (GO:0043226) and 'cellular component' (GO:0005575) and under molecular function category were 'ion binding' (GO:0043167) and 'molecular function' (GO:0003674). The results of the GO analysis are summarized in Table IV.

\section{DISCUSSION}

In the current study, we have addressed the expression profiles of the miRNA-199a-5p in the degenerated intervertebral lumbar disc disease compared to healthy controls. We have demonstrated that the fold change and relative expression of the miRNA-199a-5p was significantly higher in those with IDD when compared to traumatic normal discs. Both the fold change and relative expression of the miRNA-199a-5p was associated with Pfirrmann grade. But none of the clinical signs were associated with the expression of miRNA-199a-5p. The AUC for the miRNA-199a-5p was indicative of the moderate accuracy which can be used a biological marker for diagnosis of IDD. This is the first study to address the role of miRNA$199 a-5 p$ in IDD process.

This study was conducted to quantify the results of our previously published meta-analysis which clearly demonstrated that miR-199a-5p was upregulated in IDD. In the current experimental study, we have found that the upregulation of miR-199a-5p was associated with IDD and also correlated with clinical grades of degeneration. We have previously demonstrated that the miR-199a-5p is associated with IDD process by targeting several 4 main pathways including the ECM-receptor interaction, adherens junction, Hippo signaling pathway and endocytosis (16). Previous studies have also demonstrated that miR-199a-5p is associated with regulation of cell proliferation, motility, and angiogenesis and the target gene has been found to be the caveolin-1 (Cav-1) (9,29). In a recent study, Yan et al. found that the apoptosis of myocardiocytes in rat model of congestive heart failure is associated with upregulation of the miR-199a$5 p$ (25). Several other lines of evidence have suggested that the upregulation of miR-199a-5p is associated with Wallerian degeneration and neuronal apoptosis in patients with spinal cord injury (18), apoptosis induction and reduction in cell size in hemangiomas (21), as well as induction of autophagy in different tissue targets (22). Some other experimental studies have demonstrated that the downregulation of miR-199a-5p is associated with progression of the prostate adenocarcinoma (31), as well as increased inflammatory response by the synovial cells in patients with osteoarthritis (23).

The key step in pathogenesis of the IDD is considered the imbalance between the regeneration and degeneration of the ECM which is focused on NP cell death and apoptosis (1). The degeneration of the ECM is mediated by several enzymes including the matrix metalloproteinases (MMPs) and a disintegrin and metalloprotease with thrombospondin motifs 
(ADAMTSs) and the regulation of these enzymes are being conducted by several miRNAs $(8,20)$. In the current human study, we have demonstrated that the upregulation of miR$199 a-5 p$, is associated with IDD and also the clinical grades of degeneration based on the Pfirrmann classification. We have previously demonstrated that upregulation of miR-199a-5p is associated with ECM degeneration through ECM-receptor interaction, adherens junction and endocytosis targeting several genes the most important of which are COL6A1, COL5A1, COL1A1 and CD44 (16). All the proposed genes and pathways are involved in synthesis and production of various types of collagen in both NP and AF of the intervertebral disc $(11,15,30)$. According to these findings, it could be concluded that these genes are regulated and modulated by the miR-

Table IV: The Gene Ontology (GO) Results for the miRNA-199a-5p in IDD

\begin{tabular}{|c|c|}
\hline GO Category & $\mathbf{p}$ \\
\hline Cell cycle & 0.000101064 \\
\hline Innate immune response & 0.000193713 \\
\hline DNA metabolic process & 0.00026874 \\
\hline Post-translational protein modification & 0.000308443 \\
\hline $\begin{array}{l}\text { Positive regulation of protein insertion into mitochondrial membrane involved in apoptotic signaling } \\
\text { pathway }\end{array}$ & 0.000395602 \\
\hline Nucleobase-containing compound catabolic process & 0.000485736 \\
\hline Blood coagulation & 0.001054219 \\
\hline G2/M transition of mitotic cell cycle & 0.001101432 \\
\hline Membrane organization & 0.001125041 \\
\hline Peptidyl-threonine phosphorylation & 0.001405645 \\
\hline Mrna metabolic process & 0.001902518 \\
\hline Apoptotic signaling pathway & 0.002417346 \\
\hline Extracellular matrix organization & 0.002476532 \\
\hline Intrinsic apoptotic signaling pathway & 0.003976994 \\
\hline Cell death & 0.003976994 \\
\hline Epidermal growth factor receptor signaling pathway & 0.004237119 \\
\hline Vesicle-mediated transport & 0.00464742 \\
\hline Platelet activation & 0.008002058 \\
\hline Cellular component assembly & 0.008002058 \\
\hline Negative regulation of translation & 0.009683478 \\
\hline Antigen processing and presentation of exogenous peptide antigen via MHC class II & 0.01032818 \\
\hline Transcription, DNA-templated & 0.010563393 \\
\hline Macromolecular complex assembly & 0.010943705 \\
\hline RNA metabolic process & 0.013364541 \\
\hline Nucleotide-binding domain, leucine rich repeat containing receptor signaling pathway & 0.014479592 \\
\hline Regulation of cytoskeleton organization & 0.015736245 \\
\hline Peptidyl-serine phosphorylation & 0.016185583 \\
\hline Cell motility & 0.020583501 \\
\hline Fc-gamma receptor signaling pathway involved in phagocytosis & 0.023904308 \\
\hline Positive regulation of extrinsic apoptotic signaling pathway via death domain receptors & 0.044716545 \\
\hline
\end{tabular}


199a-5p which affects the IDD process. Further studies, should include target analysis and development of target therapy based on the findings of the current study.

The alteration in the immune profile of the intervertebral disc and the inflammatory response are also considered an important step in pathogenesis of the IDD $(4,24)$. We have also demonstrated that the miR-199a-5p upregulation is associated with increased inflammatory response through transforming growth factor (TGF)-beta signaling which in turn results in elevated IDD process. The proposed targeted genes for this pathway includes TGFBR1, THBS1, TGFB1 (16). The NP cells produce the TGF-beta as well as the fibronectin which affects the rearrangement of the ECM in patients with IDD (5). The expression and regulation of the miRNAs are also being modulated by the NP cells (13). According the recent lines of evidence, TGF- $\beta$ is a key player of IDD through induction of the matrix alterations by autocrine and paracrine routes (17). The results of our study confirms the previous findings which indicates the role of TGF-beta signaling pathway in IDD. This pathway is also regulated by miR-199a-5p. complementary studies with real time PCR are required to shed light on the issue.

We note some limitations to our study. First, we included a limited number of patients in both study groups especially the controls. The limited number of the patients included was due to the fact that we had limited resources and statistically minimum number of patients were calculated and included. However, the study had the power of $80 \%$ for detection of difference in expression profile of the miRNA-199a-5p in patients with IDD and healthy controls. Second, we did not measure the protein products of the miRNA through the western blot. The next step in our study would be performing the western blot to detect the protein changes of the IVD and to correlate the changes in protein synthesis profile with the expression profile of the miRNA-199a-5p. The other limitation was lack of luciferase-reporter assay to detect the exact target gene and to conform the results of the bioinformatics. Further studies including these miRNAs are recommended. Taking all these together, this is the first study to demonstrate the role of miRNA-199a-5p in IDD process. Further studies are currently underway to detect the exact target gene, protein products and also to determine the serum level of this miRNA as a biological marker.

\section{CONCLUSION}

The results of the current study demonstrate that the expression of the miRNA-199a-5p is increased in the degenerated lumbar intervertebral disc compared to healthy disc. The expression of the miRNA-199a-5p was also associated with the grade of the degeneration based on the Pfirrmann grading. The diagnostic accuracy of the miRNA199a-5p as a biological marker of the IDD was also found to be of moderate accuracy. The Hippo signaling pathway and the fatty acid biosynthesis were the proposed pathways through which this miRNA affects the collagen synthesis and the ECM regeneration. Further studies are required to determine the target genes and the protein synthesis target.

\section{ACKNOWLEDGMENT}

We would like to thank all the patients and their families who participated in the current study. This manuscript is the result of as thesis by Dr. Fariborz Ghaffarpasand as partial fulfillment of specialty degree in Neurosurgery being supported by a grant (No. 14413) by deputy of Research of Shiraz University of Medical Sciences. We would like to acknowledge the editorial assistance of Diba Negar Research Institute for improving the English and style of the manuscript.

\section{REFERENCES}

1. Biyani A, Andersson GB: Low back pain: Pathophysiology and management. J Am Acad Orthop Surg 12:106-115, 2004

2. Burke JG, Watson RW, McCormack D, Dowling FE, Walsh MG, Fitzpatrick JM: Intervertebral discs which cause low back pain secrete high levels of proinflammatory mediators. J Bone Joint Surg Br 84:196-201, 2002

3. Clouet J, Fusellier M, Camus A, Le Visage C, Guicheux $\mathrm{J}$ : Intervertebral disc regeneration: From cell therapy to the development of novel bioinspired endogenous repair strategies. Adv Drug Deliv Rev 2018 (Epub ahead of print)

4. Gruber HE, Norton HJ, Sun Y, Hanley EN Jr: Crystal deposits in the human intervertebral disc: Implications for disc degeneration. Spine J 7:444-450, 2007

5. Hondke S, Cabraja M, Kruger JP, Stich S, Hartwig T, Sittinger $M$, Endres M: Proliferation, Migration, and ECM formation potential of human annulus fibrosus cells is independent of degeneration status. Cartilage 2018 (Epub ahead of print)

6. Hoy D, March L, Brooks P, Blyth F, Woolf A, Bain C, Williams G, Smith E, Vos T, Barendregt J, Murray C, Burstein R, Buchbinder R: The global burden of low back pain: Estimates from the Global Burden of Disease 2010 study. Ann Rheum Dis 73:968-974, 2014

7. Hu B, Xu C, Tian Y, Shi C, Zhang Y, Deng L, Zhou H, Cao $\mathrm{P}$, Chen H, Yuan W: Inflammatory microRNA-194 and -515 attenuate the biosynthesis of chondroitin sulfate during human intervertebral disc degeneration. Oncotarget 8:4930349317, 2017

8. Lan PH, Liu ZH, Pei YJ, Wu ZG, Yu Y, Yang YF, Liu X, Che L, Ma CJ, Xie YK, Hu QJ, Wan ZY, Wang HQ: Landscape of RNAs in human lumbar disc degeneration. Oncotarget 7:63166-63176, 2016

9. Lino Cardenas CL, Henaoui IS, Courcot E, Roderburg C, Cauffiez C, Aubert S, Copin MC, Wallaert B, Glowacki F, Dewaeles E, Milosevic J, Maurizio J, Tedrow J, Marcet B, LoGuidice JM, Kaminski N, Barbry P, Luedde T, Perrais M, Mari B, Pottier N: miR-199a-5p Is upregulated during fibrogenic response to tissue injury and mediates TGFbeta-induced lung fibroblast activation by targeting caveolin-1. PLoS Genet 9:e1003291, 2013

10. Liu X, Che L, Xie YK, Hu QJ, Ma CJ, Pei YJ, Wu ZG, Liu ZH, Fan LY, Wang HQ: Noncoding RNAs in human intervertebral disc degeneration: An integrated microarray study. Genom Data 5:80, 81, 2015

11. Lv ZT, Gao ST, Cheng P, Liang S, Yu SY, Yang Q, Chen AM: Association between polymorphism rs12722 in COL5A1 and musculoskeletal soft tissue injuries: A systematic review and meta-analysis. Oncotarget 9:15365-15374, 2018 
12. Maher C, Underwood M, Buchbinder R: Non-specific low back pain. Lancet 389:736-747, 2017

13. Nerlich AG, Bachmeier BE, Boos N: Expression of fibronectin and TGF-beta1 mRNA and protein suggest altered regulation of extracellular matrix in degenerated disc tissue. Eur Spine $\mathrm{J}$ 14:17-26, 2005

14. Pfirrmann CW, Metzdorf A, Zanetti M, Hodler J, Boos N: Magnetic resonance classification of lumbar intervertebral disc degeneration. Spine (Phila Pa 1976) 26:1873-1878, 2001

15. Sato T, Takano R, Tokunaka K, Saiga K, Tomura A, Sugihara H, Hayashi T, Imamura Y, Morita M: Type VI collagen alpha1 chain polypeptide in non-triple helical form is an alternative gene product of COL6A1. J Biochem 164(2):173-181, 2018

16. Sherafatian M, Abdollahpour HR, Ghaffarpasand F, Yaghmaei $\mathrm{S}$, Azadegan M, Heidari M: MicroRNA expression profiles, target genes and pathways in intervertebral disc degeneration; a meta-analysis of three microarray studies. World Neurosurg 126:389-397, 2019

17. Specchia N, Pagnotta A, Toesca A, Greco F: Cytokines and growth factors in the protruded intervertebral disc of the lumbar spine. Eur Spine J 11:145-151, 2002

18. Tang $Y$, Ling ZM, Fu R, Li YQ, Cheng X, Song FH, Luo HX, Zhou LH: Time-specific microRNA changes during spinal motoneuron degeneration in adult rats following unilateral brachial plexus root avulsion: Ipsilateral vs. contralateral changes. BMC Neurosci 15:92, 2014

19. Vergroesen PP, Kingma I, Emanuel KS, Hoogendoorn RJ, Welting TJ, van Royen BJ, van Dieen JH, Smit TH: Mechanics and biology in intervertebral disc degeneration: A vicious circle. Osteoarthritis Cartilage 23:1057-1070, 2015

20. Wang C, Wang WJ, Yan YG, Xiang YX, Zhang J, Tang ZH, Jiang ZS: MicroRNAs: New players in intervertebral disc degeneration. Clin Chim Acta 450:333-341, 2015

21. Wang Y, Dai YX, Wang SQ, Qiu MK, Quan ZW, Liu YB, Ou JM: miR-199a-5p inhibits proliferation and induces apoptosis in hemangioma cells through targeting HIF1A. Int J Immunopathol Pharmacol 31:394632017749357, 2018
22. Wang Y, Luo J, Wang X, Yang B, Cui L: MicroRNA-199a$5 p$ induced autophagy and inhibits the pathogenesis of ankylosing spondylitis by modulating the mTOR signaling via directly targeting ras homolog enriched in brain (Rheb). Cell Physiol Biochem 42:2481-2491, 2017

23. $\mathrm{Wu} \mathrm{MH}$, Tsai $\mathrm{CH}$, Huang $\mathrm{YL}$, Fong $\mathrm{YC}$, Tang $\mathrm{CH}$ : Visfatin promotes IL-6 and TNF-alpha production in human synovial fibroblasts by repressing miR-199a-5p through ERK, p38 and JNK signaling pathways. Int J Mol Sci 19(1)pii: E190, 2018

24. Wuertz K, Vo N, Kletsas D, Boos N: Inflammatory and catabolic signalling in intervertebral discs: The roles of NF-kappaB and MAP kinases. Eur Cell Mater 23:103-119; discussion 119-120, 2012

25. Yan M, Yang S, Meng F, Zhao Z, Tian Z, Yang P: MicroRNA $199 a-5 p$ induces apoptosis by targeting JunB. Sci Rep 8:6699, 2018

26. Ying SY, Chang DC, Lin SL: The microRNA (miRNA): Overview of the RNA genes that modulate gene function. Mol Biotechnol 38:257-268, 2008

27. Ying SY, Chang DC, Lin SL: The MicroRNA. Methods Mol Biol 936:1-19, 2013

28. Yu FX, Meng Z, Plouffe SW, Guan KL: Hippo pathway regulation of gastrointestinal tissues. Annu Rev Physiol 77:201-227, 2015

29. Zeng J, Chen L, Chen B, Lu K, Belguise K, Wang X, Yi B: MicroRNA-199a-5p regulates the proliferation of pulmonary microvascular endothelial cells in hepatopulmonary syndrome. Cell Physiol Biochem 37:1289-1300, 2015

30. Zhong B, Huang D, Ma K, Deng X, Shi D, Wu F, Shao Z: Association of COL1A1 rs1800012 polymorphism with musculoskeletal degenerative diseases: A meta-analysis. Oncotarget 8:75488-75499, 2017

31. Zhong J, Huang R, Su Z, Zhang M, Xu M, Gong J, Chen N, Zeng $\mathrm{H}$, Chen X, Zhou Q: Downregulation of miR-199a-5p promotes prostate adeno-carcinoma progression through loss of its inhibition of HIF-1alpha. Oncotarget 8:8352383538, 2017 